\title{
FERROFLUIDIC PLUG FLOW HEAT TRANSFER ENHANCEMENT
}

\author{
NICOLETTE GAN JIA GUI ${ }^{1}$, CAMERON STANLEY ${ }^{1}$, NAM-TRUNG NGUYEN ${ }^{2} \&$ \\ GARY ROSENGARTEN ${ }^{1}$ \\ ${ }^{1}$ School of Engineering, Aerospace, Mechanical and Manufacturing, RMIT University, Melbourne, Australia. \\ ${ }^{2}$ Queensland Micro and Nanotechnology Centre, Griffith University, Brisbane, Australia.
}

\begin{abstract}
Overheating of power electronic devices has become a significant issue due to their continued miniaturization and increased heat flux that needs to be dissipated. Microchannel heat sinks utilising two-phase flow are capable of very high heat transfer rates and represent a possible means of cooling such devices. In this paper, we focus on two-phase liquid-liquid plug flow using water-based ferrofluid (magnetic nanofluid) plugs as the dispersed phase and silicone oil as the continuous phase. An external magnetic field was applied to generate enhanced mixing of the microfluidic flow. We show that material properties of the ferrofluid plug influence heat transfer properties of the microfluidic flow, and demonstrate that cooling performance is further enhanced by the application of an external magnetic field, which induces mixing. We also show that microchannel heat transfer using a ferrofluid is superior to that using de-ionized water as the dispersed phase for two-phase liquid-liquid plug flow.

Keywords: electronics cooling, ferrofluid, heat transfer, magnetic field, microfluidic, two-phase
\end{abstract}

\section{INTRODUCTION}

Improved cooling technologies for power electronic devices are in high demand due to the desire for greater power density and the rapid expansion of photovoltaic systems. Integrated liquid cooling provides a solution to facilitate further miniaturization and increased power density of electronic devices. However, integrated liquid cooling utilises microchannels to transport fluid, and microchannel fluid flow is characterised by laminar flow at low Reynolds numbers. These characteristics make molecular diffusion the main mode of heat transfer, which is insufficient to remove heat at a rate that is required from high performance power electronic devices. Hence, to enhance heat transfer, we introduced three 'upgrades'. The first upgrade is to improve the thermal conductivity of the working fluid. Ferrofluids are composed of small (3-15 $\mathrm{nm}$ ) particles of solid, magnetic, single-domain particles coated with a molecular layer of a dispersant or surfactant, and suspended in a liquid carrier [1]. The percentage, size and type of magnetic nanoparticle, surfactant and dispersion medium, including the method of synthesis dictates the thermophysical properties of ferrofluids, which influences the transport properties and thus, heat transfer properties of the microfluidic flow [2-5]. Thermal agitation keeps the particles suspended because of Brownian motion, and the coatings prevent the particles from sticking to each other [1].

Due to the small size of the particles, and thus increased contact surface area for heat transfer, ferrofluids offer the ability to significantly enhance heat transfer as reflected by the higher thermal conductivity it has compared to traditional cooling liquids such as de-ionized water (DIW) $[3,6,7]$. Ferrofluids have been commonly used in audio loudspeakers to improve its audio response and exhibit better power handling. They are also used as seals for rotating shafts to almost eliminate frictional losses compared to traditional mechanical seals or highspeed computer disk drives to keep out harmful dust particles and impurities. Therefore, manufacturing technologies of commercial ferrofluids are advanced and reliable. The second upgrade is to introduce rapid mixing in the ferrofluidic flow. This can be induced within the 
microchannel flow either by active or passive methods [8]. In this paper, we actively induce rapid mixing by the application of an external magnetic field using permanent magnets. The superparamagnetic nanoparticles in ferrofluids allows them to be easily manipulated by an external magnetic field, and immediately redisperses once the magnetic field is removed. The third upgrade is to adopt two-phase flow. Two-phase flow has been shown to significantly enhance heat transfer rates relative to single-phase flow by up to 500\% [9-12]. Furthermore, because liquids have higher thermal conductivity than gases, two-phase liquid-liquid flow has the potential to enhance heat transfer more effectively than two-phase gas-liquid flow [12]. Ferrofluid plugs and silicone oil are the dispersed and continuous phase respectively.

Combining these three upgrades provides the potential for the dissipation of ever increasing heat loads, which aids in the development of greater product reliability and performance of power electronic devices.

\subsection{Two-Phase Heat Transfer}

There has been no publications reporting the use of ferrofluid plugs as the dispersed phase in two-phase liquid-liquid plug flow to enhance heat transfer rates. However, there have been studies on heat transfer in two-phase liquid-liquid Taylor/slug/plug flow using silicone oil as the continuous phase, and DIW as the dispersed phase in minichannels. The fundamental concepts from these studies should be applicable to the research study with the exception of using ferrofluid plugs instead of DIW. Eain et al. [11] conducted experimental investigations examining potential heat transfer enhancements of a two-phase liquid-liquid Taylor flow regime. They studied the effects of slug length and carrier phase variations of Pd5, Dodecane and AR20 silicone oils on the local Nu. Enhancements up to $600 \%$ over conventional Poiseuille flow were observed in the fully-developed region. It was found that shorter carrier slugs approaching the channel diameter, and longer water slugs provided the greatest heat enhancement. Furthermore, it was also reported that slugs are able to reach thermal equilibrium faster, thus having shorter thermal entrance lengths as compared to continuous flow. Bandara [13] also conducted numerical and experimental studies on heat transfer and pressure drop on two-phase liquid-liquid slug flow using silicone oil and DIW. He reported having up to $400 \%$ enhancements in heat transfer compared to conventional Poiseuille flow. This gives confidence in enhancing heat transfer utilising two-phase liquid-liquid plug flow. It must be noted that studies by Liu et al. [14] and Che et al. [10] report that shorter plug lengths lead to higher heat transfer, which is in disagreement to Eain et al. [11].

\section{MATERIALS AND EXPERIMENTAL METHODS}

\subsection{Material Characterization}

\subsubsection{Particle Sizing}

It is well known that the size, shape and composition of the magnetic nanoparticles strongly influence the thermophysical profile of the ferrofluid, and thus, their transport and flow properties. Since all the diluted samples contain magnetite $\left(\mathrm{Fe}_{3} \mathrm{O}_{4}\right)$ magnetic nanoparticles, the only variable is size. Depolarized dynamic light scattering (DDLS) and transmission electron microscopy (TEM) were employed to size the particles.

\subsubsection{Transport Properties}

Viscosity and thermal conductivity are important properties for designing heat dissipation devices. These properties depend on the type and concentration of magnetic nanoparticles 
and surfactant make-up of the ferrofluid. The type and strength of external magnetic field applied also affects these properties.

\section{Viscosity}

Kinematic viscosity of the diluted ferrofluid samples was measured at $25^{\circ} \mathrm{C}$ using a capillary viscometer (Micro-Ubbelohde 531 10, SCHOTT) along with a viscosity measuring system (ViscoSystem ${ }^{\circledR}$ AVS350, SCHOTT). The viscosity measuring system records the time taken to flow past two points in the capillary viscometer. This measurement was taken three times, and the final reading is the average of the three. DIW was used as the calibration standard.

Grade N42 neodymium permanent magnets (40 x 15 x 8 mm, Aussie Magnets) were used to apply an external magnetic field to the ferrofluid. Magnetic flux, $\mathrm{B}$ was varied $(0 \leq \mathrm{B} \leq 50$ $\mathrm{mT}, \Delta \mathrm{B}=10 \mathrm{mT}$ ) by varying the offset distance between the capillary tube and the magnets.

\section{Thermal Conductivity}

Thermal conductivity of the ferrofluid samples were measured at $25^{\circ} \mathrm{C}$ using the $\mathrm{KD} 2$ Pro Thermal Property Analyzer (Decagon Devices Inc.), which is based on the transient hot-wire method. The KS-1 single needle sensor was used. The fluid sample was held in an aluminium box connected to a water bath (MB-5, Julabo), hence temperature of the sample was kept at a constant. Permanent magnets were used to apply an external magnetic field to the ferrofluids and magnetic flux was varied $(0 \leq \mathrm{B} \leq 50 \mathrm{mT}, \Delta \mathrm{B}=10 \mathrm{mT})$ by varying the offset distance between the aluminium box and the magnets.

\subsection{Dispersed Phase}

\subsubsection{Commercial Ferrofluid}

The domain detection kit consisting of EMG308, EMG408, EMG707, EMG708 and EMG807 (Domain Detection Kit, Ferrotec) ferrofluid samples were used for single-phase flow heat transfer experiments. The samples were then diluted with DIW to make diluted samples of $5 \%$ and $10 \%$ ferrofluid content with DIW (refer to Table 1).

For two-phase flow experiments, only EMG707 (Ferrotec) was used to make a diluted sample of $20 \%$ ferrofluid content with DIW. $20 \%$ dilution gives a ratio of ferrofluid:DIW as 0.2:0.8. Thermophysical properties of the diluted commercial ferrofluid sample were measured and compiled (refer to Table 1).

\subsubsection{Self-Fabricated Ferrofluids}

In order to have control and information on the fluid and surfactants, we also made our own water-based $\mathrm{Fe}_{3} \mathrm{O}_{4}$ ferrofluids. Different surfactants were used using the co-precipitation method, which forms polydisperse iron oxide nanoparticles [15]. To make about $3.75 \mathrm{~g}$ of $\mathrm{Fe}_{3} \mathrm{O}_{4}$ nanoparticles, the following material and steps were used;

1. Take $3.26 \mathrm{~g}$ of $\mathrm{FeCl}_{2} \cdot 4 \mathrm{H}_{2} \mathrm{O}$ and $8.7 \mathrm{~g}$ of $\mathrm{FeCl}_{3} \cdot 6 \mathrm{H}_{2} \mathrm{O}$ and dissolve in $380 \mathrm{~mL}$ of DIW.

2. Under vigorous stirring, slowly add $20 \mathrm{~mL}$ of $25 \% \mathrm{NH}_{3}$. The $\mathrm{pH}$ should be about 11-12.

3. Sediment the precipitate with a very strong rare earth magnet (or centrifuge for 5 minutes at $5000 \mathrm{rpm}$ ) and wash until the $\mathrm{pH}$ drops from 10 to 7.

4. Continue to sediment and precipitate until desired volume concentration of $\mathrm{Fe}_{3} \mathrm{O}_{4}$ superparamagnetic iron oxide nanoparticles (SPIONs) is obtained. 
Table 1: Measured properties of commercial diluted ferrofluid samples.

\begin{tabular}{|c|c|c|c|c|}
\hline \multirow[b]{2}{*}{ Sample } & \multirow[b]{2}{*}{$\begin{array}{l}\text { Magnetite Content } \\
\text { (\% by Volume) }\end{array}$} & \multicolumn{3}{|c|}{ Measured Properties } \\
\hline & & $\begin{array}{l}\text { Density } \\
\left(\mathrm{kg} / \mathrm{m}^{3}\right)\end{array}$ & $\begin{array}{l}\text { Dynamic } \\
\text { Viscosity } \\
(\mathrm{mPa} \cdot \mathrm{s})\end{array}$ & $\begin{array}{l}\text { Thermal } \\
\text { Conductivity } \\
(\mathrm{W} /(\mathrm{m} \cdot \mathrm{K}))\end{array}$ \\
\hline EMG308 5\% & $0.02-0.055$ & 1006.0 & 1.025 & 0.646 \\
\hline EMG308 10\% & $0.04-0.11$ & 1008.1 & 1.046 & 0.644 \\
\hline EMG408 5\% & $0.02-0.055$ & 1004.6 & 1.029 & 0.636 \\
\hline EMG408 10\% & $0.04-0.11$ & 1006.2 & 1.040 & 0.626 \\
\hline EMG707 5\% & $0.05-0.2$ & 1012.9 & 1.078 & 0.642 \\
\hline EMG707 10\% & $0.1-0.4$ & 1021.0 & 1.103 & 0.645 \\
\hline EMG707 20\% & $0.2-0.8$ & 1022.5 & 1.113 & 0.655 \\
\hline EMG708 5\% & $0.05-0.2$ & 1005.9 & 1.029 & 0.682 \\
\hline EMG708 10\% & $0.1-0.4$ & 1009.8 & 1.060 & 0.684 \\
\hline EMG807 5\% & $0.05-0.4$ & 1006.7 & 1.040 & 0.644 \\
\hline EMG807 10\% & $0.1-0.8$ & 1009.7 & 1.050 & 0.651 \\
\hline DIW & - & 1000.0 & 1.000 & 0.610 \\
\hline
\end{tabular}

To determine the volume concentration of the SPIONs, a Prussian blue assay was performed. It was found that the SPIONs had a solid volume concentration of $1.5 \%$. Before functionalizing the SPIONs, TEM was performed to check whether the SPIONs are worth functionalizing. The particle size $\left(\mathrm{d} \sim 10 \mathrm{~nm}\right.$ for $\left.\mathrm{Fe}_{3} \mathrm{O}_{4}\right)$ and coagulation (whether the particles clumped together) were the criterion of interest.

Three different surfactants were used to functionalize the fabricated SPIONs - dopamine (DPA), tetramethylammounium hydroxide (TMAH), and citric acid. The surfactants were added drop-wise to the SPIONs while swirling at the same time. All three ferrofluid types had the same volume concentration of solid magnetic nanoparticles $(\sim 1.5 \%)$ and surfactant $(\sim 10 \%)$. Only the ferrofluids fabricated with DPA and TMAH as surfactants remained stable. They were then diluted with DIW to $20 \%$ dilution, which gives a ratio of ferrofluid:DIW as 0.2:0.8. Thermophysical properties of the diluted samples were measured and compiled in the Table 2.

\subsection{Continuous Phase}

$0.65 \mathrm{cSt}$ silicone oil (PMX-200, Xiameter) with a thermal conductivity of $0.1 \mathrm{~W} /(\mathrm{m} \cdot \mathrm{K})$ was used as the continuous phase in two-phase flow heat transfer experiments.

Table 2: Measured properties of fabricated diluted ferrofluid samples

\begin{tabular}{lllll}
\hline \multirow{5}{*}{\begin{tabular}{l} 
Magnetite Content \\
\cline { 3 - 5 } Sample
\end{tabular}} & \multicolumn{3}{l}{ Measured Properties } \\
\cline { 3 - 5 } DPA Volume $)$ & Density $\left(\mathrm{kg} / \mathrm{m}^{3}\right)$ & $\begin{array}{l}\text { Dynamic } \\
\text { Viscosity }(\mathrm{mPa} \cdot \mathrm{s})\end{array}$ & $\begin{array}{l}\text { Thermal Conduc- } \\
\text { tivity }(\mathrm{W} /(\mathrm{m} \cdot \mathrm{K}))\end{array}$ \\
TMAH 20\% & $\sim 0.3$ & 1003.5 & 1.014 & 0.630 \\
& $\sim 0.3$ & 1006.2 & 1.037 & 0.686 \\
\hline
\end{tabular}




\subsection{Microchannel Fabrication}

To fabricate the microchannel, we adopted the proposed method by Saggiomo and Velders [16]. The design of the sacrificial microchannel mould was first 3D printed out of acrylonitrile butadiene styrene (ABS) with a layer thickness of $0.254 \mathrm{~mm}$ (or $254 \mu \mathrm{m}$ ) at RMIT's Advanced Manufacturing Precinct (AMP) using the uPrint SE 3D Printer (Stratasys) (see Fig. 1). The microchannel mould undergoes vapour smoothing with acetone. Following this process, optical profiling was performed using the Olympus LEXT OLS4100 3D Measuring Laser Microscope to obtain the surface roughness measurements of the untreated and treated microchannel sacrificial moulds. Two untreated (samples 1 and 2) and two treated (samples 3 and 4) sacrificial moulds were inspected. The results show that the treated sacrificial moulds are about four times smoother compared to the untreated moulds, with an approximate roughness to channel diameter ratio of 1/100. A nichrome heater coil (wire gauge 28) was then carefully wrapped around the treated sacrificial mould at the allocated location along the straight length. Thermocouples were positioned near the microchannel with the help of access ports created using additional stems in the ABS thermocouple adapter. Degassed PDMS at a mixture ratio of 10:1 was then poured into the pre-prepared glass mould over the sacrificial mould. The PDMS is left to cure in the oven at $75^{\circ} \mathrm{C}$ for $2-3$ hours. The thermocouple adapter and PDMS mould was removed, and the cured thermocouple-embedded microchannel PDMS is soaked in acetone for at least 48 hours to allow the acetone to soften the sacrificial mould. Acetone was then pushed through the inlet/outlet ports of the PDMS mould using a needleless syringe until all the sacrificial mould was fully dissolved and flushed out, creating a cavity. Finally, DIW is flushed through the cavity to clear any remaining solid particles, producing the desired microchannel.

\subsection{Flow Visualization - Enhanced Magnetic Mixing}

Fluorescein mixture - $0.05 \mathrm{~g}$ of fluorescein sodium salt (F6377-100G, Sigma-Aldrich) mixed with $20 \mathrm{~mL}$ of DIW and EMG707 20\% ferrofluid (Ferrotec) were the materials used. The 3D casted mould was used as the microfluidic chip. Single-phase flow was visualized using an inverted microscope (Eclipse TS100, Nikon) and recorded using a microscopy camera (USB 2.0 Camera, Edmund Optics). Ferrofluid and fluorescein mixture were individually introduced to the microfluidic chip using a precision syringe pump (SPM 100, SIMTech Microfluidics Foundry) via a plastic syringe (10 mL luer slip, Terumo). All connection tubes were attached to the microfluidic chip using ultraviolet (UV) curing glue (3526, Loctite). A $3.2 \mathrm{~mm}^{3}$ neodymium $(\mathrm{NdFeB})$ permanent magnet (B222, K\&J Magnetic Inc.) was used to introduce an external magnetic field. The mixture of ferrofluid and fluorescein dye was collected at the outlet collection reservoir and properly disposed of after the experiment. The magnet was placed at two different positions - magnet position 1 and magnet position 2 (see Fig. 2), to observe any difference in mixing ability due to magnetic flux strength.

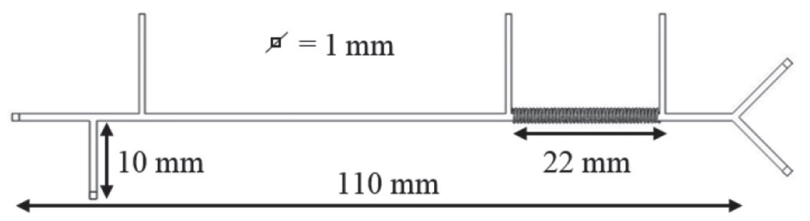

Figure 1: Microchannel sacrificial mould dimensions. 


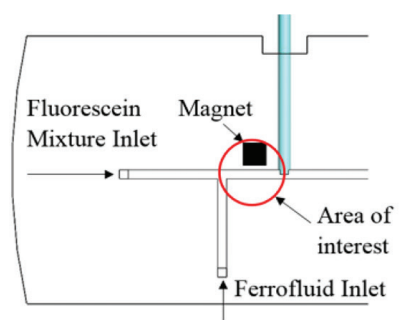

(a)

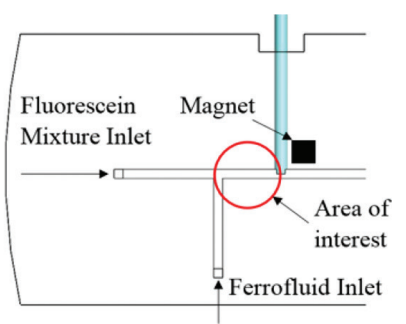

(b)

Figure 2: Enhanced magnetic mixing. (a) Magnet Position 1; (b) Magnet Position 2.

\subsection{Heat Transfer}

\subsubsection{D Casted Mould}

Eight T-type thermocouples (Teflon-insulated twisted pair solid 1/0.2 mm diameter) were embedded in the PDMS; two thermocouples embedded into the microchannel flow - one at the inlet heated area, and the other at the outlet heated area. The other six thermocouples were along the microchannel length (see Fig. 3). Small diameter thermocouple wires were used to minimise conduction losses. Temperature was recorded using a temperature data logger (TC08, Pico Technology). The temperature measurement system was calibrated against a traceable platinum resistance thermometer (PRT) (P795, Dostmann) in a uniform temperature water bath with an immersion circulator (MPC-E, Huber).

\subsubsection{Two-Phase Flow}

Three different ferrofluid samples - EMG707 20\%, TMAH $20 \%$ and DPA 20\% were used as the dispersed phase, while $0.65 \mathrm{cSt}$ silicone oil was used as the continuous phase. Prior to recording data using ferrofluidic plugs, DIW was used as the dispersed phase to calibrate the obtained results.

\subsubsection{Nusselt Number Calculation}

Nusselt number, $\mathrm{Nu}$, is expressed as

$$
\mathrm{Nu}=\frac{\mathrm{hD}_{\mathrm{h}}}{\kappa}
$$

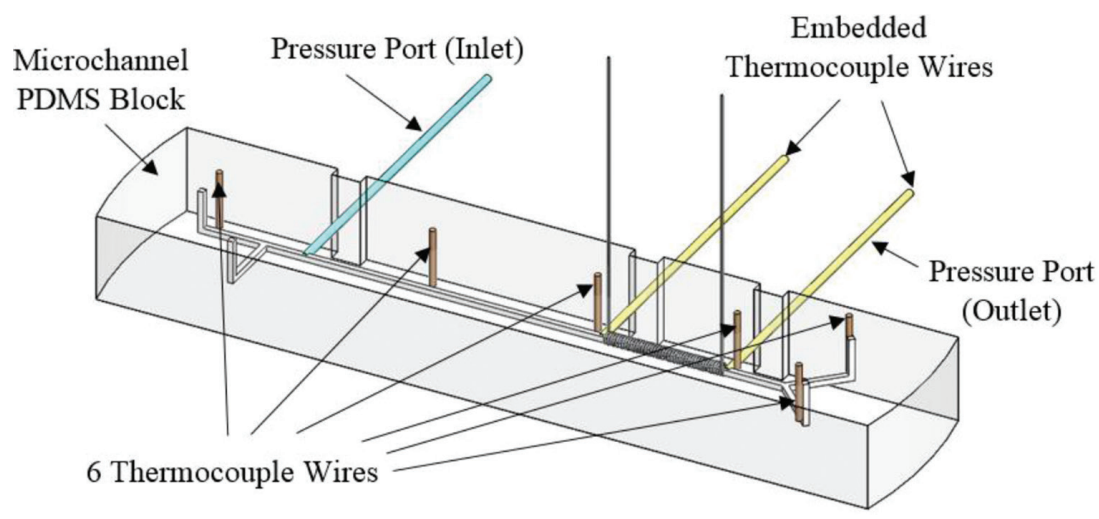

Figure 3: Embedded thermocouples in microchannel flow (Isometric View) 
where $\mathrm{D}_{\mathrm{h}}$ represents the characteristic length or hydraulic diameter in $\mathrm{m}$, and $\kappa$ is the thermal conductivity of the fluid in $\mathrm{W} /(\mathrm{m} \cdot \mathrm{K})$. The convective heat transfer coefficient of the flow, $\mathrm{h}$ in $\mathrm{W} /\left(\mathrm{m}^{2} \cdot \mathrm{K}\right)$, is expressed as

$$
\mathrm{h}=\frac{\mathrm{q}_{\mathrm{f}}}{\left(\mathrm{T}_{\mathrm{w}, \mathrm{avg}}-\mathrm{T}_{\mathrm{f}, \mathrm{avg}}\right)}
$$

where $\mathrm{q}_{\mathrm{f}}$ is the heat flux in $\mathrm{W} / \mathrm{m}^{2}, \mathrm{~T}_{\mathrm{w}, \mathrm{avg}}$ is the average wall temperature difference $\left(\mathrm{T}_{\mathrm{w}, \text { out }}+\right.$ $\left.\mathrm{T}_{\mathrm{w}, \text { in }}\right) / 2$ in $\mathrm{K}$, and $\mathrm{T}_{\mathrm{avg}}$ is the average fluid temperature difference $\left(\mathrm{T}_{\text {out }}+\mathrm{T}_{\mathrm{in}}\right) / 2$ in $\mathrm{K}$. Here, $\mathrm{q}_{\mathrm{f}}$ can be calculated as

$$
\mathrm{q}_{\mathrm{f}}=\frac{\mathrm{Q} \rho \mathrm{c}_{\mathrm{p}}\left(\mathrm{T}_{\text {out }}-\mathrm{T}_{\mathrm{in}}\right)}{\mathrm{A}}
$$

where Q is the volumetric flow rate in $\mathrm{m}^{3} / \mathrm{s}, \rho$ is density of the fluid in $\mathrm{kg} / \mathrm{m}^{3}, \mathrm{c}_{\mathrm{p}}$ is the specific heat capacity of the fluid in $\mathrm{J} /(\mathrm{kg} \cdot \mathrm{K}),\left(\mathrm{T}_{\text {out }}-\mathrm{T}_{\text {in }}\right)$ is the average fluid temperature difference in $\mathrm{K}$, and $\mathrm{A}$ is the area in contact with the fluid in $\mathrm{m}^{2}$.

As the solid volume fraction of magnetic nanoparticles is estimated to have an average of $0.03-0.4 \%$ depending on the sample, $\rho$ and $c_{p}$ for ferrofluid was assumed to have about the same value as the carrier fluid (DIW).

For the experiments, $N u$ was scaled in accordance with DIW, where the theoretical value of Nusselt number is 3.61 for a square cross-section microchannel.

\section{RESULTS AND DISCUSSION}

\subsection{Material Characterization}

\subsubsection{Particle Sizing}

The average particle size for all ferrofluid samples that were obtained performing DDLS was between $6-8 \mathrm{~nm}$. The reliability of these results was verified by performing TEM on the samples, which resulted in an average particle size of 10-15 nm. The particle sizes obtained from TEM are slightly larger than those obtained from DDLS. This is due to the evaporation under ambient atmosphere of the solution during sample preparation, where the nanoparticles are deposited onto strong carbon grids (GSCu200C-50), spurring the nanoparticles towards further aggregation due to the elevating free energy at the grain boundary [5].

\subsubsection{Transport Properties}

It was observed that viscosity increases linearly with increase in magnetic flux strength at room temperature (RTP), and thermal conductivity increases exponentially with increase in magnetic flux strength (see Fig. 4).

\subsection{Flow Visualization}

\subsubsection{Single-Phase Enhanced Magnetic Mixing}

In the absence of a magnetic field, a 1:1 ratio of ferrofluid:fluorescein mixture needs to be achieved (see Fig. 5). Fluorescein mixture and the ferrofluid were introduced at a flow rate of $800 \mu \mathrm{L} / \mathrm{min}$ and $50 \mu \mathrm{L} / \mathrm{min}$, respectively. Without a magnet, very slight mixing of the ferrofluid and fluorescein mixture occurred via molecular diffusion. However, with the application 


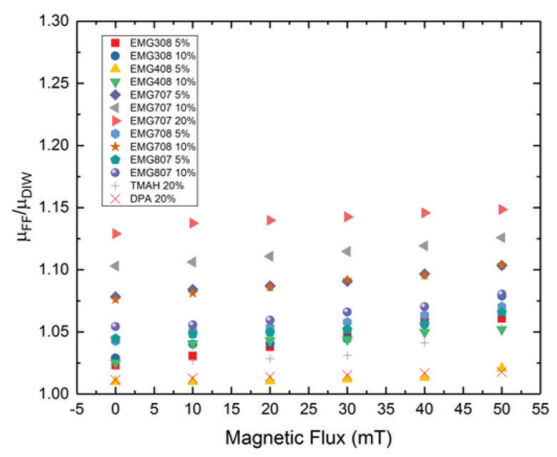

(a)

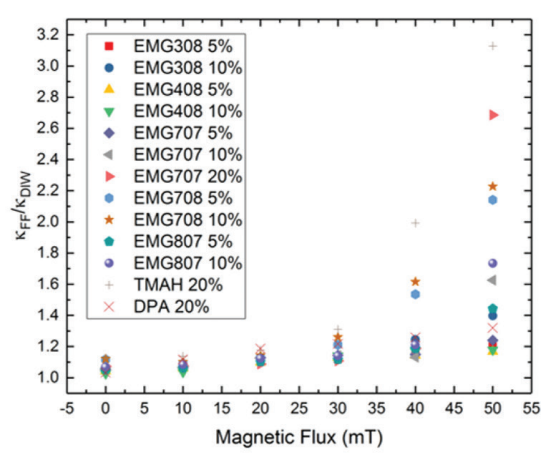

(b)

Figure 4: Transport properties of ferrofluids (with magnetic field) (a) $\mu_{\mathrm{FF}} / \mu_{\mathrm{DIW}}$; (b) $\kappa_{\mathrm{FF}} / \kappa_{\mathrm{DIW}}$.

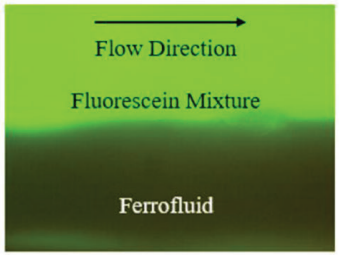

(a)

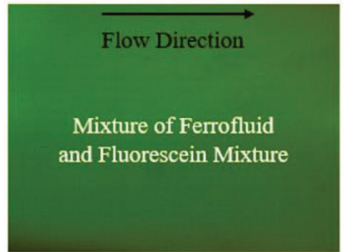

(b)

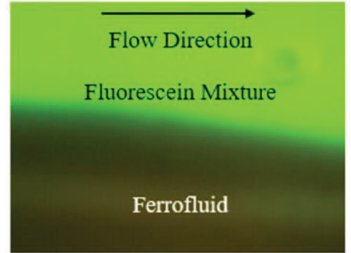

(c)

Figure 5: Single-phase flow visualization - enhanced magnetic mixing results. (a) No magnetic field; (b) Magnet position 1; (c) Magnet position 2.

of a magnetic field, it was observed that mixing was greatly enhanced. At magnet position 1 (see Fig. 2), mixing occurred rapidly as observed by the fluorescein mixture mixing with the ferrofluid and filling the entire channel (see Fig. 5). At magnet position 2, because the magnetic field is further away from the area of interest, magnetic flux applied to the area is therefore reduced (see Fig. 5). Hence, less mixing is observed. It can thus be concluded that mixing is enhanced with increased magnetic flux.

\subsection{Heat Transfer}

\subsubsection{DIW Calibration}

As a benchmark, against which to compare the heat transfer enhancement provided by the ferrofluids, the heat transfer enhancement of two-phase flow using DIW as the dispersed phase and $0.65 \mathrm{cSt}$ silicone oil to single-phase DIW was measured. Results showed an approximate doubling $(200 \%)$ of heat transfer of the two-phase compared to single-phase flow.

\subsubsection{Single-Phase Heat Transfer Enhancement (No Magnetic Field)}

EMG708 5\% recorded the highest heat transfer enhancement of 116\% improvement compared to DIW (see Fig. 6). This large improvement can be attributed to the induced magnetic flux of $6 \mathrm{mT}$ produced by the nichrome heater coil. EMG708 10\% has a higher percentage of magnetic nanoparticles, which should theoretically result in higher heat transfer rates. However, EMG708 $10 \%$ records a lower $\mathrm{Nu}$ compared to EMG708 5\%. 


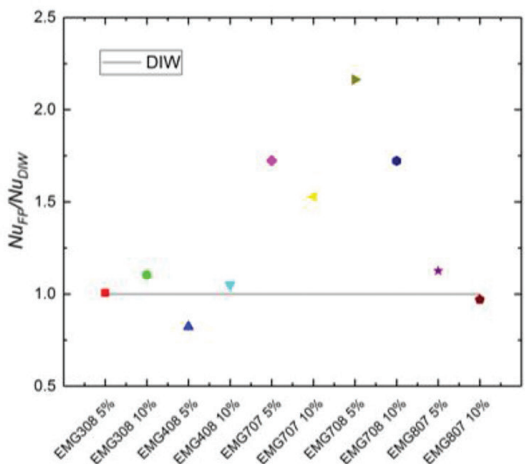

(a)

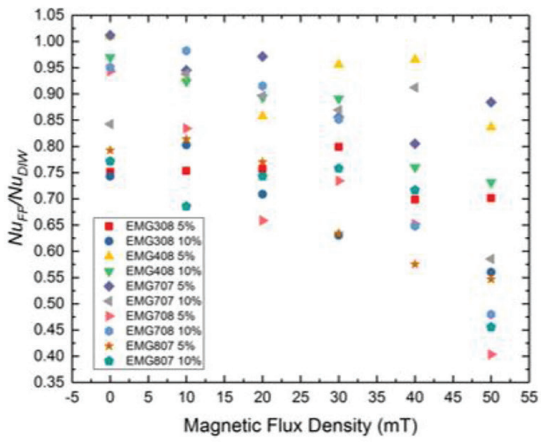

(b)

Figure 6: Single-phase flow $N u_{F F} / N u_{D I W}$ Results, Re = 11.6. (a) No magnetic field; (b) With magnetic field.

\subsubsection{Single-Phase Heat Transfer Enhancement (With Magnetic Field)}

Magnetic field strengths of 10, 20,30, 40 and $50 \mathrm{mT}$ were applied to the fluid flow at the heated section. Generally, it was observed that $N u$ decreases with increasing magnetic field strength for all diluted ferrofluid samples (see Fig. 6). This could be attributed to the solid magnetic nanoparticles getting attracted to, and at high enough magnetic fluxes pinned in place against, the wall of the channel under the action of the magnetic field. The removal of the nanoparticles from the carrier fluid reduces the effective thermal conductivity of the mixture. This in turn is detrimental to heat transfer at the area where magnetic flux is applied and high magnetic fluxes.

\subsubsection{Two-Phase Ferrofluidic Plug Flow Heat Transfer Enhancement (No Magnetic Field)}

Using ferrofluid plugs as the dispersed phase, the heat transfer rate increased (at low Re) compared to using DIW as the dispersed phase (see Fig. 7). It can also be observed that commercial ferrofluid EMG707 20\% yielded the highest heat transfer enhancement, followed by TMAH $20 \%$ then DPA 20\% in the absence of an external magnetic field. This then shows an almost three times enhancement of heat transfer rate compared to DIW only at low $\operatorname{Re}(\operatorname{Re}=2.32)$.

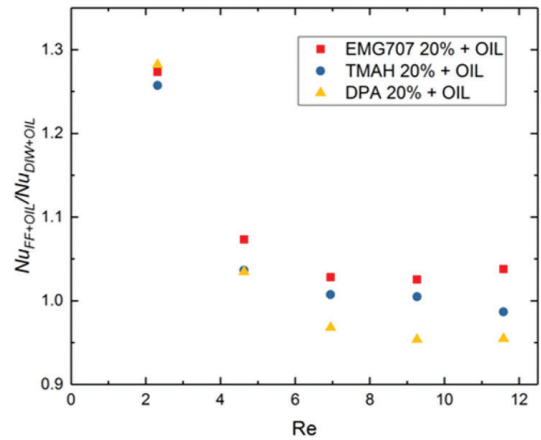

(a)

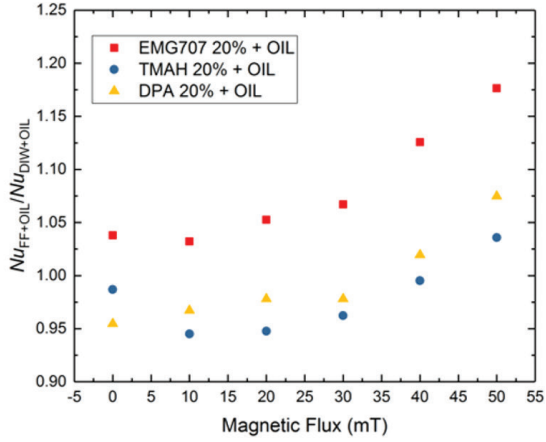

(b)

Figure 7: $N u_{\mathrm{FF}+\mathrm{OIL}} / N u_{\text {DIW+OIL }}$ Results. (a) No magnetic field; (b) With magnetic field @ Re = 11.6. 


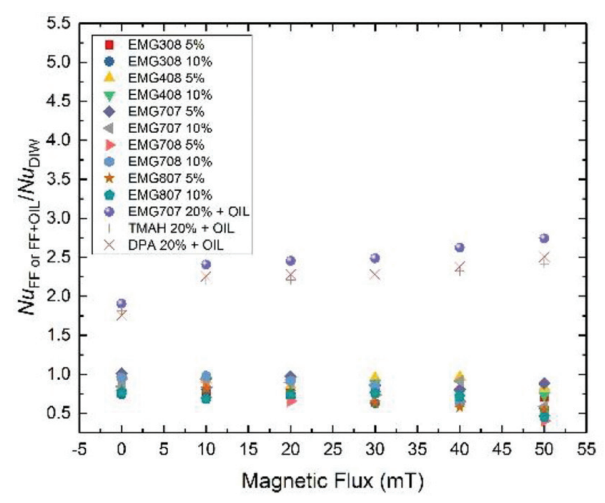

Figure 8: Comparison of Single-phase and two-phase heat transfer enhancement (with magnetic field), $\operatorname{Re}=11.6$

Heat transfer may only be enhanced at low Re because the plugs may have detached from the channel wall with increased flow rates. This is explained by Bandara [13] where plug length decreases in size with increased Re. We will visualize this experiment in future.

\subsubsection{Two-Phase Ferrofluidic Heat Transfer Enhancement (With Magnetic Field)}

Under the influence of an external magnetic field, there is improved heat transfer enhancement compared to the case when no external magnetic field was applied (see Fig. 7). It was also observed that heat transfer is enhanced with increased magnetic flux strength. This is different from single-phase flow heat transfer results previously obtained, where heat transfer rate decreases under the influence of an external magnetic field (see Fig. 8). This could be because the solid magnetic nanoparticles do not come out of solution and get stuck to the channel wall under the influence of an external magnetic field with the presence of the oil phase.

\section{CONCLUSIONS AND RECOMMENDATIONS}

Two-phase liquid-liquid plug flow showed higher heat transfer rates if DIW was replaced by a ferrofluid as the dispersed phase. Without an external magnetic field, heat transfer rate of ferrofluidic plug flow is about the same as when DIW is used as the dispersed phase, except at low Re. In general, two-phase liquid-liquid plug flow approximately doubles heat transfer rate compared to single-phase flow. We also observed that heat transfer rates are different for different ferrofluid types. Also, with the application of an external magnetic field, heat transfer rates for two-phase liquid-liquid plug flow were enhanced. We self-fabricated ferrofluids with the same magnetic nanoparticle type, and coated the nanoparticles with two different surfactants - dopamine (DPA) and tetramethylammonium hydroxide (TMAH). We found that in the absence of an external magnetic field, both DPA and TMAH coated ferrofluids exhibited similar heat transfer rate enhancement. However, in the presence of an external magnetic field, DPA coated ferrofluid recorded slightly higher heat transfer rate enhancement compared to TMAH coated ferrofluid. All in all, two-phase liquid-liquid plug flow using ferrofluid plugs as the dispersed phase, under an external magnetic field, yields higher heat transfer rates compared to using DIW as the dispersed phase.

Future works will include pressure drop studies comparing the increase in pressure drop $(\Delta \mathrm{P})$ due to two-phase liquid-liquid plug flow as opposed to single-phase flow. The aim is to maximize $N u / \Delta \mathrm{P}$. 


\section{ACKNOWLEDGEMENTS}

We thank David Dunstan, Calum Kinnear, and RMIT's MNRF, RMMF and AMP for assistance, Mark Greaves from the CSIRO Manufacturing Electron Microscopy, Digital Imaging and Surface Analysis Facility within the Characterisation Group for his contribution to the optical profiling experiment, and the Australian Research Council for funding this research (linkage project ID LP150100153).

\section{REFERENCES}

[1] Rosensweig, R.E., Ferrohydrodynamics, Dover edition ed. Mineola, New York: Dover Publications, Inc., 1997.

[2] Abareshi, M., Goharshadi, E.K., Zebarjad, S.M., Fadafan, H.K. \& Youssefi, A., Fabrication, characterization and measurement of thermal conductivity of $\mathrm{Fe} 3 \mathrm{O} 4$ nanofluids. Journal of Magnetism and Magnetic Materials, 322, pp. 3895-3901, 2010. https://doi.org/10.1016/j.jmmm.2010.08.016

[3] Alsaady, M., Fu, R., Li, B., Boukhanouf, R. \& Yan, Y., Thermo-physical properties and thermo-magnetic convection of ferrofluid. Applied Thermal Engineering, 88, pp. 14-21, 2015. https://doi.org/10.1016/j.applthermaleng.2014.09.087

[4] Ghasemi, E., Mirhabibi, A. \& Edrissi, M., Synthesis and rheological properties of an iron oxide ferrofluid. Journal of Magnetism and Magnetic Materials, 320, pp. 2635-2639, 2008. https://doi.org/10.1016/j.jmmm.2008.05.036

[5] Jiang, W. \& Wang, L., Monodisperse magnetite nanofluids: Synthesis, aggregation, and thermal conductivity. Journal of Applied Physics, 108, 2010. https://doi.org/10.1063/1.3518045

[6] Nkurikiyimfura, I., Wang, Y. \& Pan, Z., Heat transfer enhancement by magnetic nanofluids: a review. Renewable and Sustainable Energy Reviews, 21, pp. 548-561, 2013. https://doi.org/10.1016/j.rser.2012.12.039

[7] Li, Q., Xuan, Y. \& Wang, J., Experimental investigations on transport properties of magnetic fluids. Experimental Thermal and Fluid Science, 30, pp. 109-116, 2005. https://doi.org/10.1016/j.expthermflusci.2005.03.021

[8] Che, Z., Nguyen, N.T. \& Wong, T.N., Analysis of chaotic mixing in plugs moving in meandering microchannels. Physical Review E, 84, p. 066309, 2011. https://doi.org/10.1103/physreve.84.066309

[9] Nguyen, N.T. \& Wereley, S.T., Fundamentals and applications of microfluidics, Second ed.: Artech House, 2006.

[10] Che, Z., Wong, T.N. \& Nguyen, N.-T., Heat transfer enhancement by recirculating flow within liquid plugs in microchannels. International Journal of Heat and Mass Transfer, 55, pp. 1947-1956, 2012. https://doi.org/10.1016/j.ijheatmasstransfer.2011.11.050

[11] Eain, M.M.G., Egan, V. \& Punch, J., Local Nusselt number enhancements in liquid-liquid Taylor flows. International Journal of Heat and Mass Transfer, 80, pp. 85-97, 2014. https://doi.org/10.1016/j.ijheatmasstransfer.2014.09.009

[12] Bandara, T., Nguyen, N.-T. \& Rosengarten, G., Slug flow heat transfer without phase change in microchannels: a review. Chemical Engineering Science, 126, pp. 283-295, 2015.

https://doi.org/10.1016/j.ces.2014.12.007 
[13] Bandara, P.M.T., Heat transfer enhancement in microchannels with liquid-liquid slug flow. Doctor of Philosophy, RMIT University, 2015.

[14] Liu, J., Tan, S.-H., Yap, Y.F., Ng, M.Y. \& Nguyen, N.-T., Numerical and experimental investigations of the formation process of ferrofluid droplets. Microfluidics and Nanofluidics, 11, pp. 177-187, 2011. https://doi.org/10.1007/s10404-011-0784-7

[15] Lu, A.-H., Salabas, E.L. \& Schüth, F., Magnetic nanoparticles: synthesis, protection, functionalization, and application. Angewandte Chemie International Edition, 46, pp. 1222-1244, 2007. https://doi.org/10.1002/anie.200602866

[16] Saggiomo, V. \& Velders, A.H., Simple 3D printed scaffold-removal method for the fabrication of intricate microfluidic devices. Materials Views, Advanced Science, 2, pp. 1-5, 2015. 